\title{
OPEN Enhancing near-infrared photoluminescence from single-walled carbon nanotubes by defect-engineering using benzoyl peroxide
}

Lukasz Przypis $^{1}$, Maciej Krzywiecki², Yoshiaki Niidome ${ }^{3}$, Haruka Aoki ${ }^{3}$, Tomohiro Shiraki $^{3}$ \& Dawid Janas ${ }^{1 \bowtie}$

Single-walled carbon nanotubes (SWCNTs) have been modified with ester groups using typical organic radical chemistry. Consequently, traps for mobile excitons have been created, which enhanced the optical properties of the material. The proposed methodology combines the benefits of mainstream approaches to create luminescent defects in SWCNTs while it simultaneously avoids their limitations. A step change was achieved when the aqueous medium was abandoned. The selection of an appropriate organic solvent enabled much more facile modification of SWCNTs. The presented technique is quick and versatile as it can engage numerous reactants to tune the light emission capabilities of SWCNTs. Importantly, it can also utilize SWCNTs sorted by chirality using conjugated polymers to enhance their light emission capabilities. Such differentiation is conducted in organic solvents, so monochiral SWCNT can be directly functionalized using the demonstrated concept in the same medium without the need to redisperse the material in water.

Since carbon nanotubes (CNTs) were popularized in the early 1990s, many research groups have provided evidence for their extraordinary electrical ${ }^{1}$, thermal ${ }^{2}$, and optical ${ }^{3}$ nature. One of the areas which offers particular opportunities for semiconducting single-walled CNTs (s-SWCNTs) is the field of photonics owing to the presence of bandgap in these materials. As a result, they can fluoresce ${ }^{4}$ over a wide spectral range from visible to the near-infrared ${ }^{5}$ attracting a considerable share of attention to these nanostructures. Unfortunately, studies on this topic revealed that pristine SWCNTs have a low photoluminescence quantum yield (PLQY), estimated at 1.0\% in liquid ${ }^{6}$ and about $0.1 \%$ in films ${ }^{7}$.

It was recently found that slight functionalization of the material can notably enhance this property ${ }^{8}$. The introduction of $\mathrm{sp}^{3}$ defects (evident by the emergence of $\mathrm{E}_{11}{ }^{*}$ signatures) creates mobile exciton traps of appropriate energy levels, facilitating radiative recombination ${ }^{9-11}$, thereby markedly boosting the s-SWCNT PLQY ${ }^{10}$. To attain such performance, s-SWCNTs are modified by doping with oxygen ${ }^{12,13}$, or they are grafted with aryl or alkyl derivatives. The protocols of SWCNT arylation commonly involve reactions with aryldiazonium salts or $^{10,14,15}$ diazoethers (arenediazoates) ${ }^{14,16}$, whereas alkylation techniques ${ }^{17}$, are generally based on adaptations of Billups-Birch reduction protocols ${ }^{18}$.

Despite the effectiveness of these routines, most of them are limited to water dispersions or water/polar solvent mixtures. Employing aqueous media for such processing is particularly inexpedient in the light of recent milestones achieved on the front of SWCNTs sorting ${ }^{19}$. It was shown that certain conjugated-polymers soluble in organic solvents enable isolation of SWCNTs of particular electrical character or even chirality. Given that polymer-wrapped SWCNTs are compatible with organic solvents, it is highly desirable to develop an effective $\mathrm{sp}^{3}$ functionalization method dedicated to SWCNT dispersions in such media. To tackle this problem, Zaumseil and colleagues devised a concept of using diazonium salts outside of the aqueous environment ${ }^{20}$. They utilized crown ether as a phase-transfer reagent to realize the first effective diazonium salts functionalization of SWCNTs

${ }^{1}$ Department of Organic Chemistry, Bioorganic Chemistry and Biotechnology, Silesian University of Technology, B. Krzywoustego 4, 44-100 Gliwice, Poland. 'Institute of Physics-CSE, Silesian University of Technology, Konarskiego 22B, 44-100 Gliwice, Poland. ${ }^{3}$ Department of Applied Chemistry, Graduate School of Engineering, Kyushu University, 744 Motooka, Nishi-ku, Fukuoka 819-0395, Japan. ${ }^{\circledR}$ email: Dawid.Janas@polsl.pl 
in the toluene/acetonitrile mixture. Successful modification of monochiral SWCNTs dispersed with conjugated polymers was confirmed after $16 \mathrm{~h}$ of reaction in darkness.

In parallel, grafting of SWCNTs with radicals received a particular share of attention because it can be accomplished at the reduced time, as indicated by Maeda ${ }^{21}$ and Weisman groups ${ }^{13}$. Maeda et al. investigated the radical functionalization of SWCNTs under sonication of the SDBS-dispersion of SWCNTs in water ${ }^{21}$. The authors postulated that under these conditions, hydroxyl radicals and hydrogen peroxide are generated, which grafted the SWCNT sidewall to give $\mathrm{E}_{11}{ }^{*}$ and $\mathrm{E}_{11}{ }^{{ }^{*}}$ peaks. Furthermore, Weisman and co-workers demonstrated effective functionalization of SWCNTs using sodium hypochlorite under UV illumination. Oxygen defects were added in less than $1 \mathrm{~min}^{13}$. What is more, as recently shown, fluorescent defects can be implanted in SWCNTs by employing photoexcited aromatic compounds ${ }^{22}$. Strong $\mathrm{E}_{11}{ }^{*}$ and $\mathrm{E}_{11}{ }^{2^{*}}$ bands emerge rapidly, and their intensity peaks within a few minutes. These strategies show the possibility of efficient, controllable, and rapid functionalization of SWCNTs with radicals without causing collateral damage. We were inspired by these results and decided to explore if the defect chemistry can be done not in the water, wherein it suffers from the reactive nature hydroxyl radical, but in other media ${ }^{23}$.

Herein, we describe our strategy of functionalization of SWCNTs using radical organic reagents in toluene. The aim of this approach was motivated by several reasons. Firstly, we wanted to provide appropriate conditions for controllable defect implantation. For this, we used an organic solvent instead of water, thereby eliminating potential pathways for generating aggressive radicals such as $\cdot \mathrm{OH}$. Their addition is often hard to control, and hence it results in deterioration of the optical properties of the SWCNTs. Secondly, several techniques of isolation of SWCNTs of particular chirality were recently devised for organic solvents, which employ polymer surfactants ${ }^{24,25}$. It is essential to develop dedicated modification strategies for non-aqueous environments because these polymer-harvested SWCNTs are almost always insoluble in water. Direct grafting of sorted SWCNTs without the need to conduct a tedious surfactant-exchange process to make them water-compatible would be very advantageous. Lastly, by redesigning the functionalization tactic, it may be possible to significantly speed up the procedure compared with other mainstream techniques, most of which require hours ${ }^{20}$ or days ${ }^{10,15}$. To tackle these issues, we introduced luminescent $\mathrm{sp}^{3}$ defects directly to polymer-wrapped $(6,5)$ SWCNTs in toluene by exposing them to benzoyl peroxide (BPO). Upon reaching $100^{\circ} \mathrm{C}$, the functionalization is completed in $1 \mathrm{~h}$ and results in strong $\mathrm{E}_{11}{ }^{*}(1156 \mathrm{~nm})$ and $\mathrm{E}_{11}{ }^{{ }^{-}}(1280 \mathrm{~nm})$ light emission signatures. Investigation of the possible type of introduced functional group gave strong evidence that a $\mathrm{C}-\mathrm{O}$ bond between SWCNT and the substituent is formed for the first time, creating the previously unreported type of trap for mobile excitons.

\section{Results and discussion}

We started the study by analyzing the composition of the starting material: (6,5)-enriched CoMoCAT SWCNTs (Fig. 2). Water and toluene dispersions facilitated by sodium cholate (SC) as well as poly(9,9-dioctylfluorenyl2,7-diyl) and bipyridine copolymer (PFO-Bpy), respectively, were analyzed.

The parent material in water was predominantly made of $(6,5)$ species, as expected, but other chiralities were also detected. The enclosed absorption spectrum of SWCNT aqueous dispersion of SC illustrated that $(6,4)$, $(7,5),(7,6),(8,3),(8,4),(9,1)$, and $(9,2)$ species were also present (Fig. 1a, blue curve), which was supported by the corresponding 2D PLE mapping (Fig. S1).

Recently, it was reported that upon the combination of raw SWCNTs with a copolymer of poly(9,9-dioctylfluorenyl-2,7-diyl) and bipyridine (PFO-Bpy), (PFO-Bpy, instead of SC) $(6,5)$ SWCNTs can be preferentially extracted to toluene ${ }^{20,26}$. We decided to employ this approach because, in addition to chiral-selectivity of PFO-Bpy in toluene, it was an appropriate medium to solubilize organic radical precursors at concentrations on the order of a few grams per liter ${ }^{24}$. The extraction of $(6,5)$ SWCNTs was successful judging by the presence of sharp $E_{11}$ and $\mathrm{E}_{22}$ optical transitions in the absorption spectrum (Fig. 1a, grey curve) as well as the absence of signatures from other SWCNT chiralities in the 2D PLE map (Fig. 1b) at the characteristic wavelengths. Only trace amounts of $(6,4)$ and $(9,1)$ SWCNTs were detected in the absorption spectra, as reported previously for a similar separation routine using PFO-Bpy ${ }^{20}$. We deduced that the selected conjugated polymer exhibits selectivity for SWCNTs of particular diameter distribution because these three SWCNT types had a similar size. Furthermore, the $\mathrm{E}_{11}$ peak of $(6,5)$ SWCNTs was found at a relatively long wavelength $(997 \mathrm{~nm})$, which followed previous findings for toluene-based dispersions of CNTs of $(6,5)$ type $^{20}$. For water-based dispersions, wherein typical surfactants are employed, the $\mathrm{E}_{11}$ peak of these species is usually located between $980^{27}$ and $990 \mathrm{~nm}^{13}$. Therefore, besides solvent effects, the observed redshift proved that PFO-Bpy copolymer was bound tightly to the surface of the highlighted SWCNT types ${ }^{28,29}$, explaining its high differentiation selectivity.

Our method for creating luminescent $\mathrm{sp}^{3}$ defects in polymer-wrapped SWNTs relied on the addition of preformed organic radical reagents to $(6,5)$ SWCNTs in toluene. First, we evaluated four typical sources of radicals employed in organic chemistry: azobisisobutyronitrile (AIBN), $N$-iodosuccinimide (NIS), meta-chloroperoxybenzoic acid ( $m \mathrm{CPBA})$, and benzoyl peroxide (BPO). 2D PLE maps of the dispersions after the reactions carried out for $1 \mathrm{~h}$ at $100{ }^{\circ} \mathrm{C}$ are given in Fig. 2. The results provided evidence that the functionalization was successful only in the case of BPO. For this compound, a faint $\mathrm{E}_{11}{ }^{*}$ defect peak was detected at $1156 \mathrm{~nm}$ in addition to the inherent $\mathrm{E}_{11}$ signature. Functionalization attempts using other radical reagents did not produce the desired modification.

The organic radicals which we tested can be divided into two types. According to the way they are initiated, they work either by homolytic or heterolytic bond cleavage. AIBN and BPO start the radical reaction by the homolytic breaking of the bond, whereas $m \mathrm{CPBA}$ and NIS by heterolytic. In the case of homolytic dissociation, it is possible to characterize the reaction by a specific parameter s. decomposition rate $k_{d}\left[\mathrm{~s}^{-1}\right]$.

At the temperature of our experiment $\left(100^{\circ} \mathrm{C}\right)$, the values of these parameters for AIBN and BPO are $1.5 \times 10^{-3}$ and $5.0 \times 10^{-4} \mathrm{~s}^{-1}$, respectively, as reported for benzene ${ }^{30}$. In light of the foregoing, we believe that the 
(a)

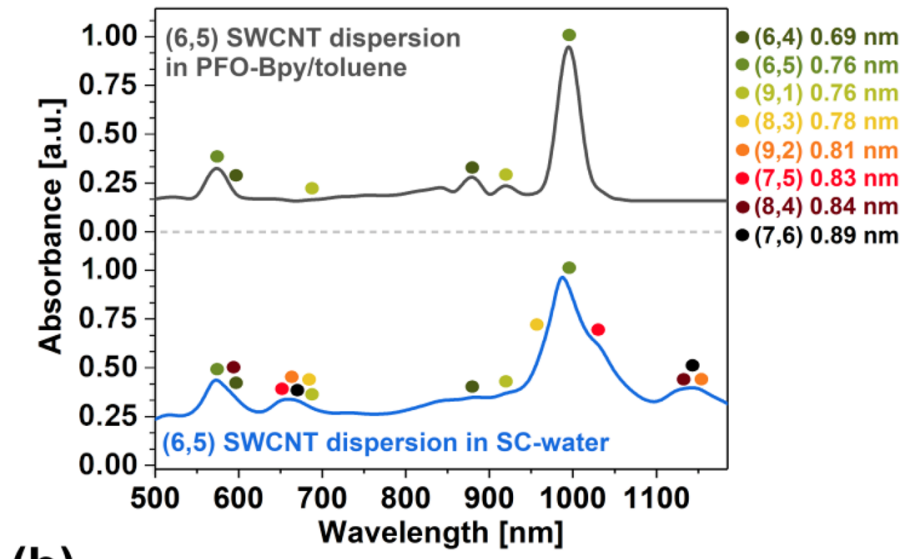

(b)

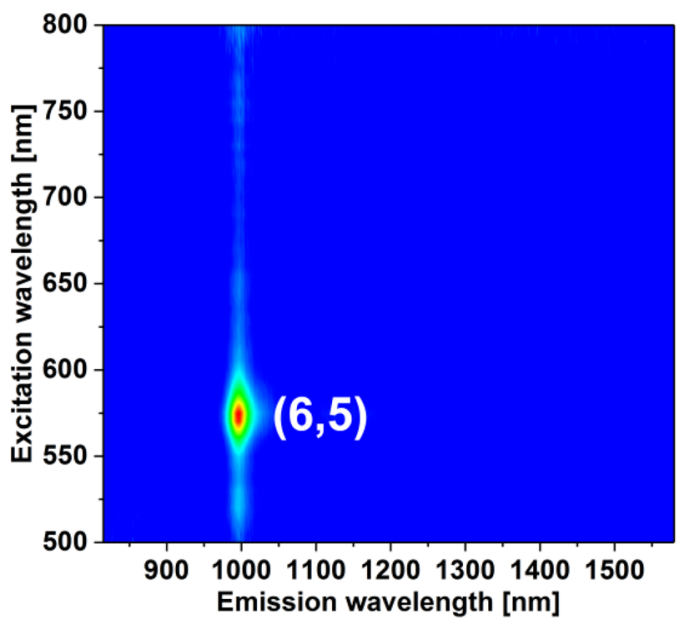

Figure 1. Characterization of the parent material. (a) Absorption spectra of (6,5)-enriched SWCNTs dispersed in $2 \%$ sodium cholate aqueous solution or PFO-Bpy/toluene solution. (b) 2D PLE map of the (6,5)-enriched SWCNT dispersion prepared by PFO-Bpy in toluene.

desired functionalization occurs when the radical forms at the optimum rate. Too slow pace does not promote the generation of the reactive species at considerable speed, whereas too fast causes self-termination of created radicals. We hypothesize that is the reason why the application of AIBN was unsuccessful. Comparing the decomposition rates shows that AIBN generates radicals three times faster than BPO under the same conditions, so they cannot be used effectively for grafting.

Furthermore, NIS and $m$ CPBA have a different radical nature. Firstly, NIS is an ambiguous reagent, and depending on the reaction conditions, it can play the role of an electrophile or radical reservoir. For NIS to induce a radical pathway, a trigger such as light or sufficiently high temperature ${ }^{31,32}$ is required. It is not uncommon to employ UV illumination or temperatures above $150^{\circ} \mathrm{C}$ to initiate the radical cascade. That may justify why it was not successful for the functionalization of SWCNTs under the thermal conditions of this study (only $100^{\circ} \mathrm{C}$ ). The stimulus was not strong enough to release the organic radicals from NIS. Secondly, $m$ CPBA is a radical reagent that is typically induced by acid-base treatment. In this case, appropriate conditions for the radical generation were not established in our system too, which explains the observed lack of reactivity of $m$ CPBA with SWCNTs. Since just BPO was successful in the explored parameter space, it was employed for further experiments.

An essential practical note is that toluene not only facilitated the dissolution of polymer-wrapped SWCNTs, but it also offered a proper reaction route when BPO was utilized. The functionalization was excessive when we tried an analogous functionalization attempt using SWCNT aqueous dispersion and BPO. In this case, the material lost light-emission capabilities altogether (Fig. S2). The action of BPO in water resulted in the generation of an abundance of aggressive hydroxyl radicals, which readily attached to the SWCNT side wall and disrupted the optical properties of the material. Conversely, when toluene was selected as a medium, it enabled the controllable addition of the organic group onto the SWCNT surface due to the favorable decomposition pathway of BPO.

We then studied the impact of the reaction time on the ability of SWCNT to luminescence (Fig. 3). As the time of the reaction was increased from 15 to $60 \mathrm{~min}$, the $\mathrm{E}_{11}{ }^{*}$ defect peak became brighter. What is more, its position shifted from 1156 to $1160 \mathrm{~nm}$. Two conclusions could be made at this point. Firstly, the selected concentration of $100 \mu \mathrm{g} / \mathrm{mL}$ for BPO was insufficient to reach optimum brightness at the specified time. No signs 
(a)

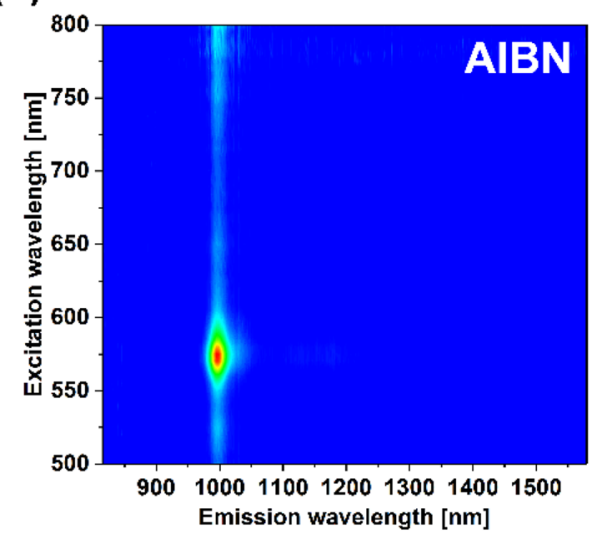

(c)

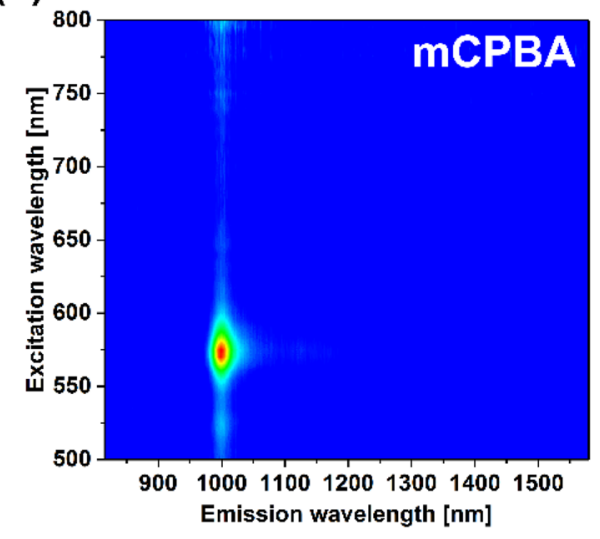

(b)

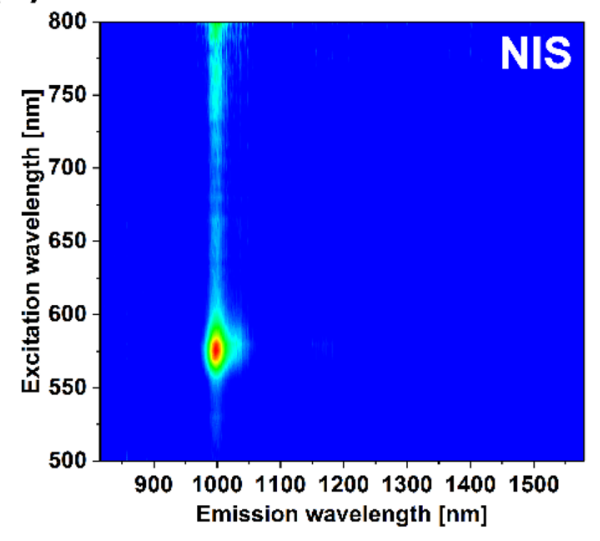

(d)

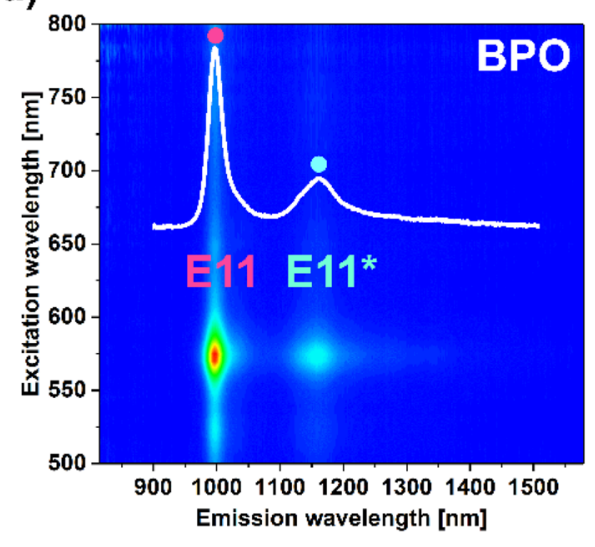

Figure 2. 2D PLE maps after treatment of the (6,5)-enriched SWCNT toluene dispersion with various radicals (a) AIBN, (b) NIS, (c) mCPBA, and (d) BPO. The inset shows a single PL spectrum collected at $575 \mathrm{~nm}$ excitation wavelength extracted from the corresponding map.

of photoluminescence quenching could be detected after $60 \mathrm{~min}$; therefore, either longer time or higher concentration of the radical compound should be employed. Secondly, the origins of the observed $\mathrm{E}_{11}{ }^{*}$ shift could not be ascribed with certainty since the shape of the spectrum was only modified to a small extent under these conditions.

To study the phenomenon in greater detail, we decided to conduct functionalization over a wide BPO concentration range from 1 to $1200 \mu \mathrm{g} / \mathrm{mL}$ (Fig. 4a). We observed a clear dependence of the concentration of radicals on the optical properties of the material. Up to $600 \mu \mathrm{g} / \mathrm{mL}$ of BPO, the intensity of the defect peak $\mathrm{E}_{11}{ }^{*}$ increased, and then it started to decrease. Exceeding this concentration threshold made the material over-functionalized, and hence the PL was quenched. Surplus of defects in the SWCNTs promoted non-radiative recombination of excitons, thereby hampering the light emission capabilities of the material.

Closer investigation of the acquired 2D PLE maps showed a notable presence of the deep trap states $\mathrm{E}_{11}{ }^{*}$ resulting from different arrangements of the grafted functional groups on the surface of the SWCNT ${ }^{5,33,34}$. The tail of the $\mathrm{E}_{11}{ }^{*}$ signature extended up to about $1550 \mathrm{~nm}$ (Fig. 5a). Because of that, despite the low maximum intensity of this peak (barely distinguishable in 2D PLE maps), its relative share in the PL properties was quite large. Under the optimum conditions of $600 \mu \mathrm{g} / \mathrm{mL}$ of BPO, the integrated intensity of $\mathrm{E}_{11}{ }^{{ }^{-}}$was almost equal to that of $\mathrm{E}_{11}{ }^{*}$ (Fig. $4 \mathrm{~b}$ ). This finding suggests that this reaction system gave many possible arrangements of functional groups on the SWCNT sidewall. Please refer to Fig. S3 to see how the areas of individual peaks $\mathrm{E}_{11}$, $\mathrm{E}_{11}{ }^{*}$, and $\mathrm{E}_{11}{ }^{*-}$ were resolved.

Nevertheless, since the absorption spectrum stayed intact after functionalization, we concluded that the functionalization degree after the treatment was low (Fig. S4). Moreover, the modification was accompanied by the increase in the $\mathrm{D} / \mathrm{G}^{+}$ratio from 0.043 to 0.131 (Fig. S4b,c), which stayed within the expected range of introduced disorder established by other reports ${ }^{8}$. Regarding the $\mathrm{E}_{11}{ }^{*} / \mathrm{E}_{11}$ ratio, the $\mathrm{E}_{11}{ }^{*}$ defect-peak was up to twice as strong as the inherent $\mathrm{E}_{11}$ signature upon modifying the surface. Such an effect was attained even in the absence of electron-withdrawing functional groups on the aryl ring (Fig. 5b). Had we introduced them to the radical precursor, the ratio would probably be higher, as reported for a similar SWCNT grafting approach ${ }^{20}$. The twofold enhancement was nonetheless appreciable. Furthermore, once the concentration of BPO was exceeded beyond $600 \mu \mathrm{g} / \mathrm{mL}$, the $\mathrm{E}_{11}{ }^{*} / \mathrm{E}_{11}$ ratio rapidly declined. The same behavior was noted when comparing the combined $\mathrm{E}_{11}{ }^{*}+\mathrm{E}_{11}{ }^{{ }^{*}}$ intensities against that of $\mathrm{E}_{11}$ (Fig. $5 \mathrm{c}$ ). 
(a)

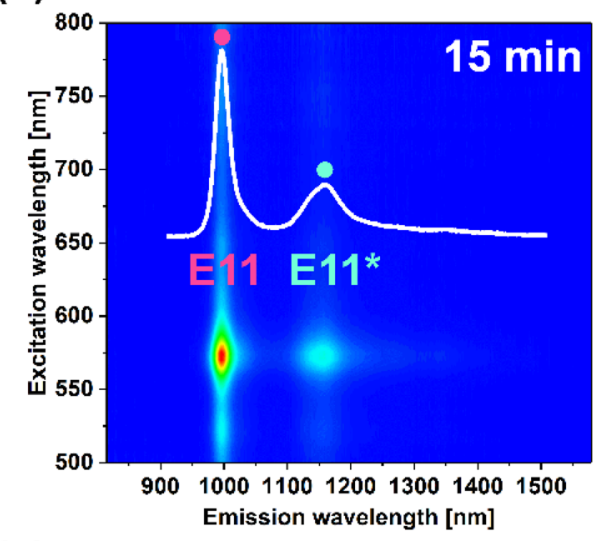

(c)

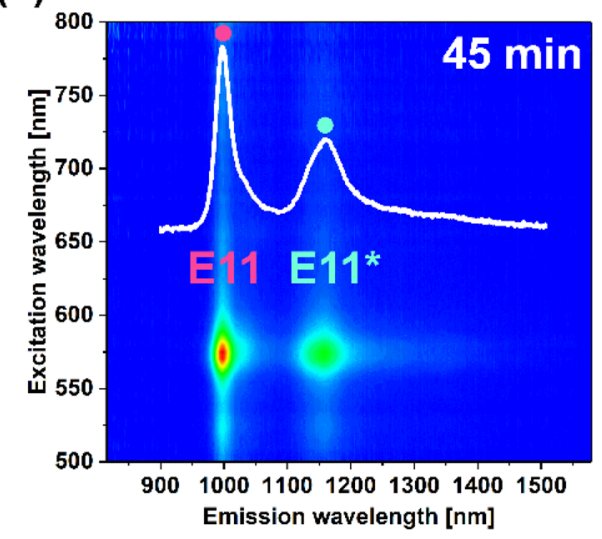

(b)

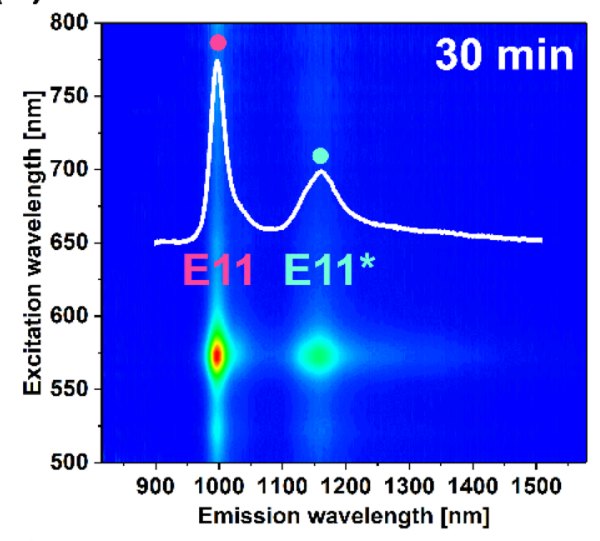

(d)

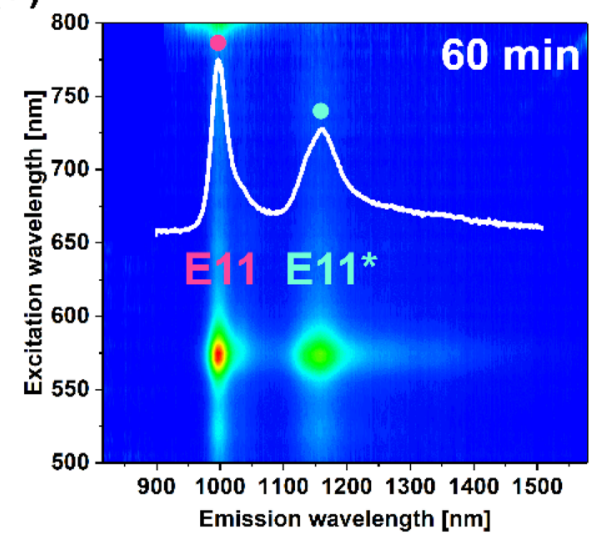

Figure 3. 2D PLE maps of the (6,5)-enriched SWCNT dispersion treated with BPO in toluene at $100 \mu \mathrm{g} / \mathrm{mL}$ at $100{ }^{\circ} \mathrm{C}$ for various times (a) $15 \mathrm{~min}$, (b) $30 \mathrm{~min}$, (c) $45 \mathrm{~min}$, and (d) $60 \mathrm{~min}$. The inset shows single PL spectra collected at $575 \mathrm{~nm}$ excitation wavelength extracted from the corresponding maps.

Lastly, the position of $\mathrm{E}_{11}$ and $\mathrm{E}_{11}{ }^{*}$ peaks was linearly dependent on the degree of functionalization (Fig. $5 \mathrm{~d}$ ). The latter peak, however, was more sensitive than the former one. The $\mathrm{E}_{11}{ }^{*}$ defect-peak was redshifted by $12 \mathrm{~nm}$ (from 1156 to $1168 \mathrm{~nm}$ ), whereas the inherent $E_{11}$ signature moved by just $5 \mathrm{~nm}(997-1004 \mathrm{~nm}$ ) as the concentration of employed BPO was increased. In other studies, the position of these peaks was invariant of reactant concentration $^{35}$, or these peaks moved in the opposite direction (away ${ }^{36}$ and towards ${ }^{20}$ each other). Our results contrasted with the previously reported work, so we suspected a new type of SWCNT functionalization.

We first noted that the new PL peak arose from the modification of SWCNTs, not from BPO in PFO-Bpy/ toluene solution as their combination did not give any peaks in the PLE map in the absence of SWCNTs (Fig. S5). The lack of $\mathrm{E}_{11}{ }^{*}$ and $\mathrm{E}_{11}{ }^{*}$ signals in that PLE map confirmed that recorded signatures came indisputably from the interaction of BPO with SWCNTs. Secondly, the position of the defect peak was strongly red-shifted from that of $O$-doped $(6,5)$-SWCNTs (1156 nm vs. $1120 \mathrm{~nm}^{37}$ ), which suggested that oxidation of SWCNTs by BPO was an unlikely cause for the observed effect.

To understand this phenomenon, we considered three independent reaction pathways (Fig. 6). In every case, in the first step, it was assumed that BPO thermally dissociated to benzoyloxy radicals. Then, the benzoyloxy radicals could react with SWCNTs directly to create previously unreported ester modification (Pathway A). In the second possible mechanism, benzoyloxy radicals could rearrange to phenyl radicals upon the expulsion of $\mathrm{CO}_{2}$ to give phenyl-modified SWCNTs (Pathway B). The position of the defect PL peak in this case defects should be in the same spectral range ca. $1160 \mathrm{~nm}$ as in recent a contribution demonstrating grafting of SWCNTs by diazonium salts in toluene ${ }^{20}$. Since we detected the $\mathrm{E}_{11}{ }^{*}$ peak maximum in this range, this reaction route could not be dismissed. The last proposed possibility (Pathway $\mathrm{C}$ ) involved the formation of benzyl radicals from the reaction of phenyl or benzoyloxy radical with toluene. In such a case, the benzyl derivative of SWCNTs would be formed. Unfortunately, the position of the defect peak of such chemical arrangement in toluene is not available in the literature as well, so we could not eliminate this possibility either. However, Maeda and co-workers showed that SWCNTs could be grafted with benzyl groups by reductive alkylation. Dispersions of modified SWCNTs prepared this way gave $\mathrm{E}_{11}{ }^{*}$ signature at about $1100 \mathrm{~nm}$, which corresponded to a Stokes shift of ca. $125 \mathrm{~nm}^{38}$. In our case, the Stokes shift was about $160 \mathrm{~nm}$, so even considering solvent and polymer-wrapping effects, the last proposed pathway was less likely.

To work out the functionalization mechanism and the type of functional group anchored on the SWCNT surface, we had to conduct additional experiments. If the first synthetic route was correct (Pathway A), we 
(a)
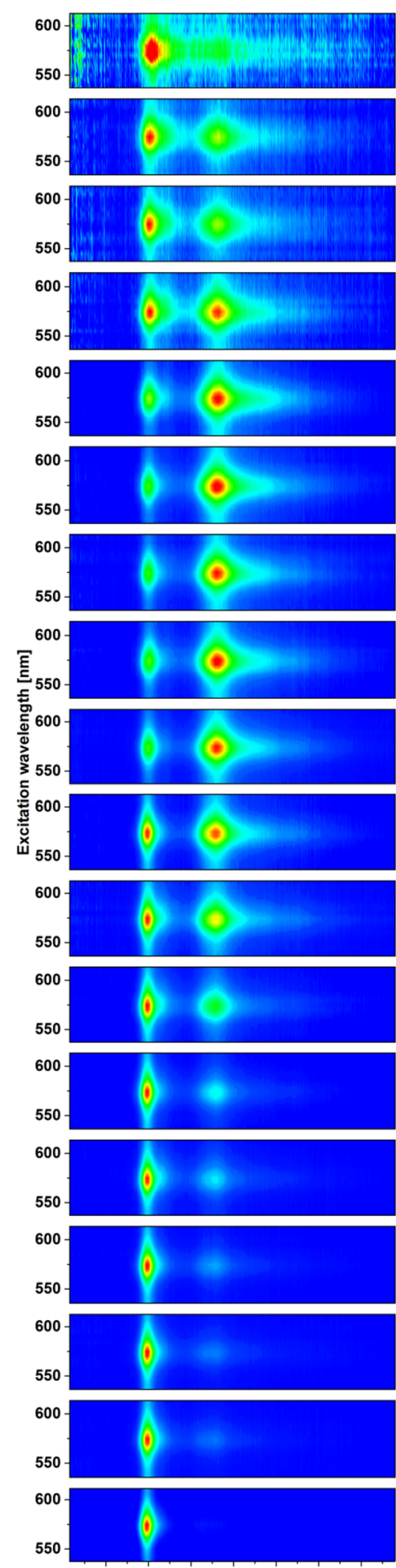

900100011001200130014001500 Emission wavelength [nm] (b)

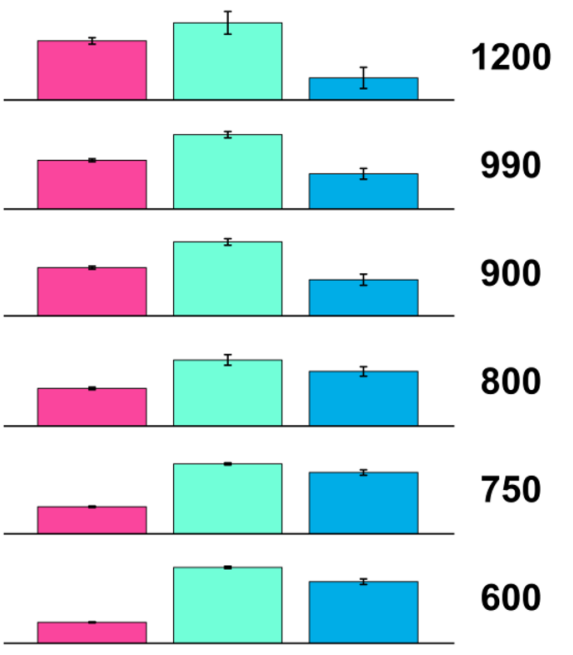

575

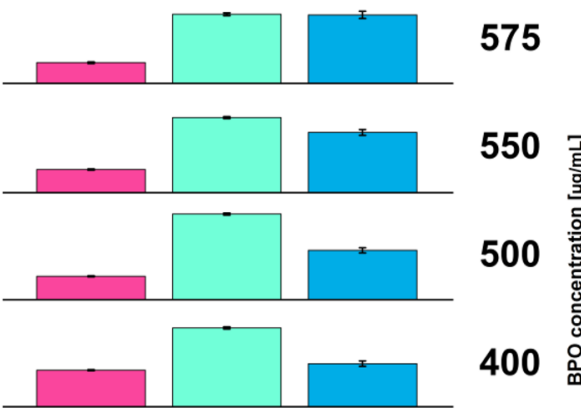

250

100

80

50

25

10

1

0

Figure 4. (a) $2 \mathrm{D}$ PLE maps of the (6,5)-enriched SWCNT dispersion treated with various BPO concentrations in toluene at $100{ }^{\circ} \mathrm{C}$ for $1 \mathrm{~h}$. (b) deconvolution of the relative intensities of the constituting $\mathrm{E}_{11}, \mathrm{E}_{11}{ }^{*}$, and $\mathrm{E}_{11}{ }^{*}$ peaks. 
(a)

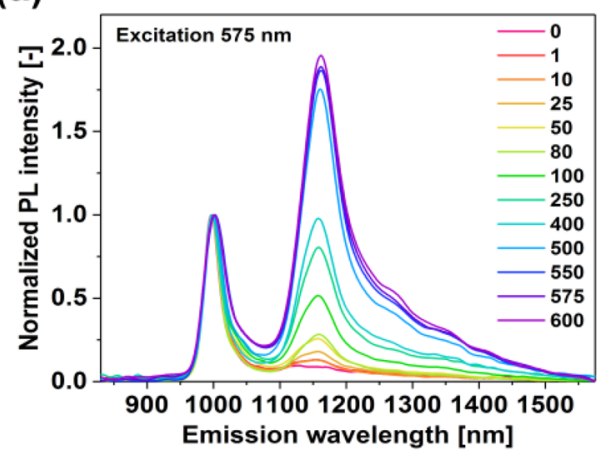

(c)

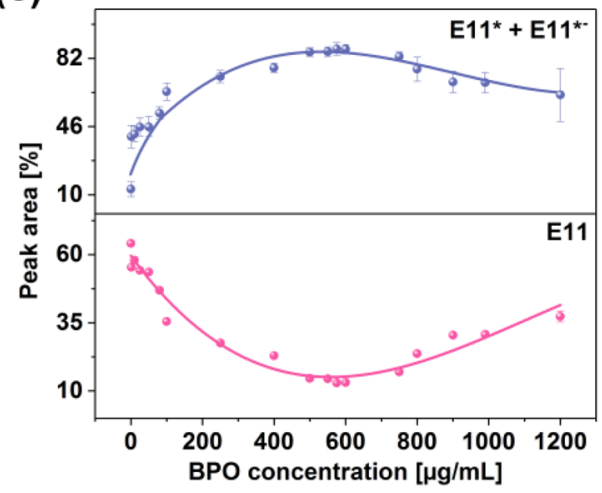

(b)

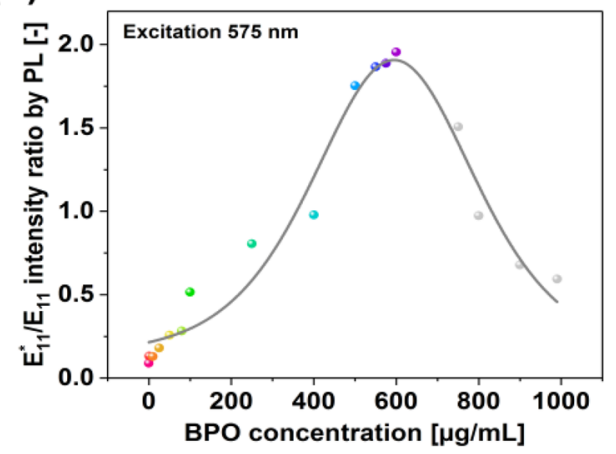

(d)

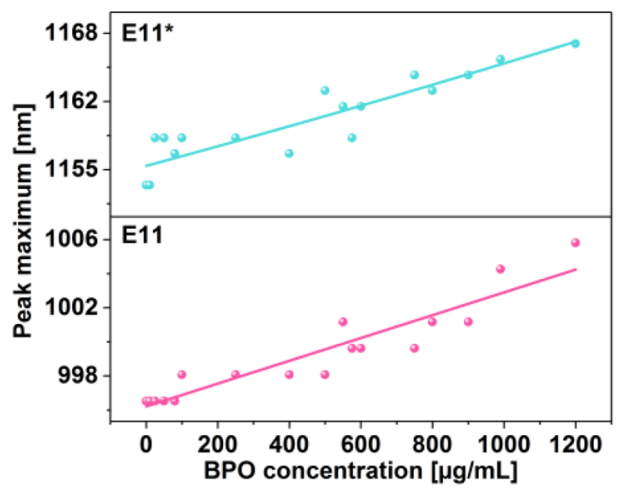

Figure 5. (a) Single PL spectra of SWCNTs collected at $575 \mathrm{~nm}$ excitation as a function of employed BPO concentration, (b) calculated ratios of $\mathrm{E}_{11}{ }^{*} / \mathrm{E}_{11}$ intensities as a function of employed $\mathrm{BPO}$ concentration, (c) the intensity of $\mathrm{E}_{11}$ and $\mathrm{E}_{11}{ }^{*}+\mathrm{E}_{11}{ }^{{ }^{*}-}$ peaks as a function of employed $\mathrm{BPO}$ concentration, and (d) position of maxima of $\mathrm{E}_{11}$ and $\mathrm{E}_{11}$ peaks as a function of employed $\mathrm{BPO}$ concentration.

could expect that the ester bond between the aryl group and SWCNT could be dissociated by hydrolysis to give corresponding alcohol and carboxylic acid under basic conditions (Figs. 7, 8). This process, in turn, should change the spectral characteristics of the $\mathrm{E}_{11}{ }^{*}$ signal. Such a reaction could not occur for the other two possible functionalization types (Pathways B and C) because the aryl groups in both these cases were attached with nonlabile C-C bonds.

It was challenging to select an appropriate base for toluene because most of them were sparingly soluble in this medium. Therefore, we decided to use ammonium hydroxide in methanol as a co-solvent for SWCNT toluene dispersion. We witnessed rapid spectral response after the treatment of the modified SWCNTs (Fig. 8a) with the base solution at room temperature after just $15 \mathrm{~min}$ (Fig. 8b). The suppression of the $\mathrm{E}_{11}{ }^{*}$ signal was observed at $1156 \mathrm{~nm}$, and additionally, a new signal at $1306 \mathrm{~nm}$ of comparable intensity was recorded. The created signature resembled the $\mathrm{E}_{11^{2 *}}$ peak caused by the proximal modification of the SWCNTs ${ }^{15}$. Moreover, the $\mathrm{E}_{11}$ signature was restored.

The results gave us more certainty that ester bonds indeed connected substituents to the SWCNT sidewall. Once these bonds were hydrolyzed, the aryl groups were released from the SWCNTs. Simultaneously, hydroxyl moieties took their positions on the SWCNT surface following the well-established mechanism of ester hydrolysis. This reasoning could explain the observed changes to the optical properties of the material gauged by $2 \mathrm{D}$ PLE. It has to be noted that such an outcome simultaneously eliminated the possibility of the functionalization to proceed via the phenyl and benzyl radical routes (Pathways B and C) because a simple base treatment could not detach these groups from the SWCNT surface.

For complete verification of our hypothesis, we executed additional control experiments. First, we checked the stability of the product after chemical transformation. The saponification reaction proceeds irreversibly, so we measured PL from the sample after $24 \mathrm{~h}$. The same outcome was reached (Fig. 8c), which supported the theory. We also validated whether the expected organic group was attached to the SWCNT sidewall to rule out the possibility of implantation of some sort of an inorganic oxygen functionality by BPO (a well-known oxidizing agent). For this, we employed an analogous BPO reactant labeled by the trifluoromethyl group. The SWCNT dispersion in toluene was reacted with bis[4-(trifluoromethyl)benzoyl] peroxide $\left(\mathrm{CF}_{3}-\mathrm{BPO}\right)$, which, if the hypothesis was correct, should produce ester modified SWCNTs (labeled with fluorine) along with certain amounts of trifluoromethylbenzene as a by-product. The side-product was preferentially removed by prolonged heating as its boiling point was lower than that of toluene used as a solvent. Once it was evaporated, we were confident that it would not interfere with the chemical analysis. The modified SWCNTs were investigated by XPS afterward (Fig. 9). 


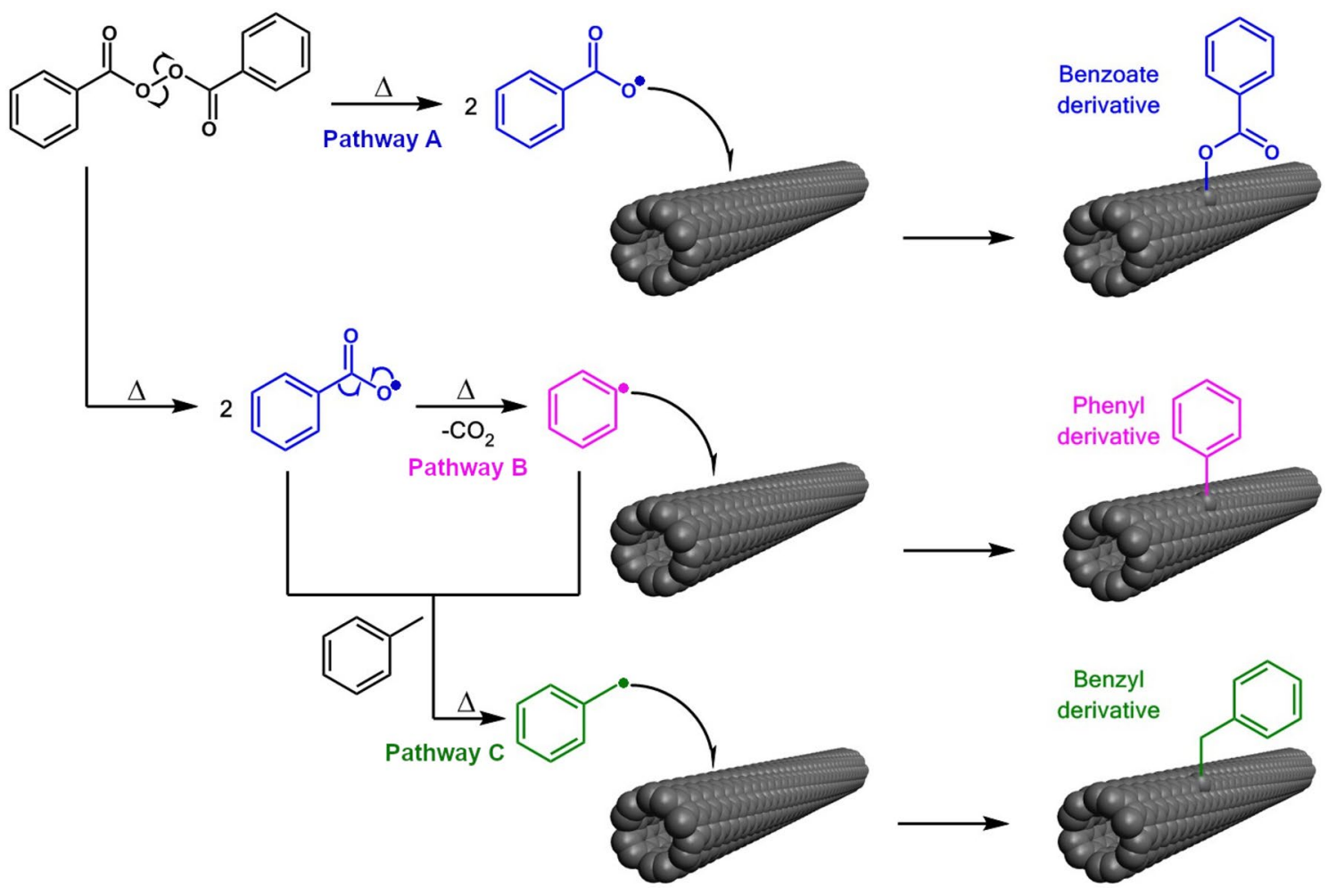

Figure 6. Proposed possible mechanisms of SWCNT functionalization by BPO in toluene, leading to ester, aryl, and arylalkyl derivatives, respectively. PFO-Bpy is not shown on the surface of SWCNTs for the sake of clarity.
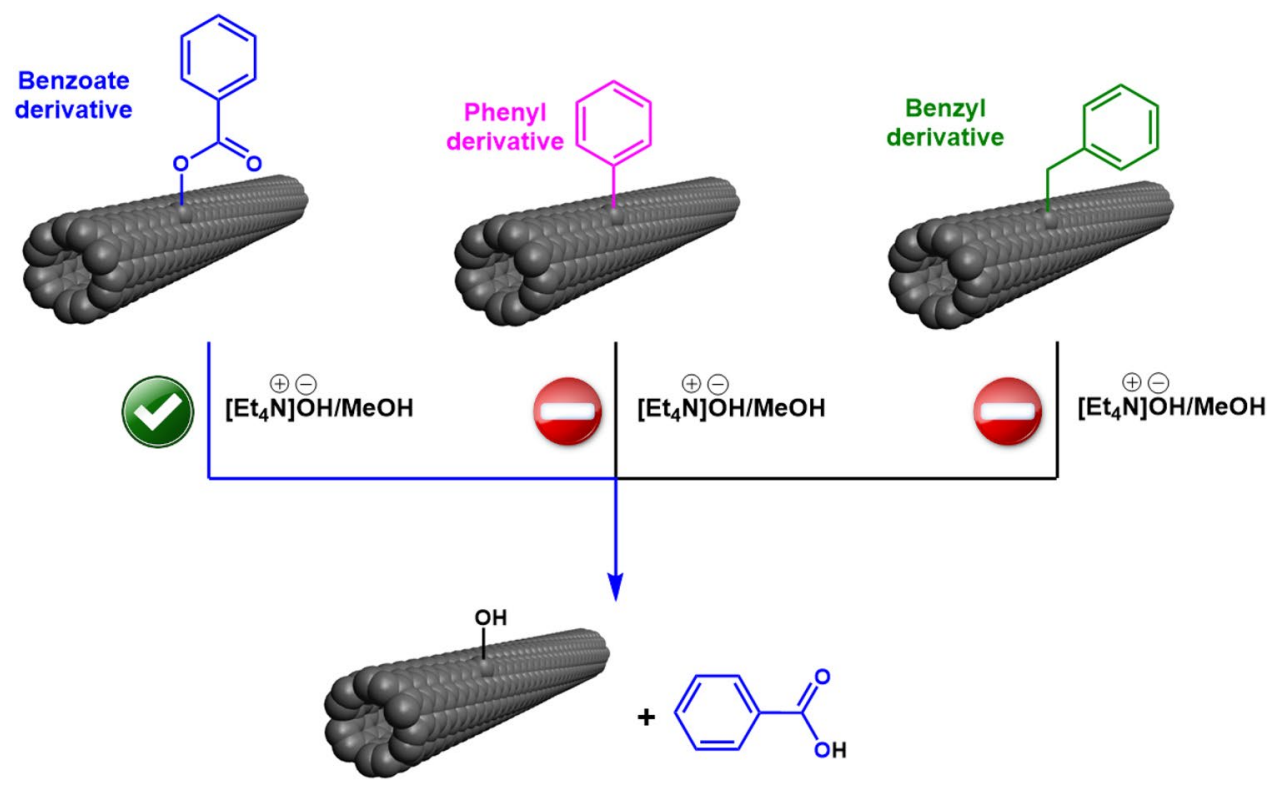

Figure 7. Basic hydrolysis of possible functional groups grafted onto the SWCNT surface by BPO in toluene. PFO-Bpy is not shown on the surface of SWCNTs for the sake of clarity.

A strong signal of fluorine in the range expected for organic fluorine was detected (Fig. 9a); therefore, we concluded that the desired functional group was successfully implanted. The low intensity of the $\mathrm{CF}_{3} \mathrm{compo}^{-}$ nent expected in the $\mathrm{C} 1 \mathrm{~s}$ region could be rationalized by substantial $\mathrm{C}-\mathrm{F}$ bond degradation by the impact of $\mathrm{X}$-radiation ${ }^{39}$. To prove the presence of fluorine in the final material, the F1s signal was recorded before any other part and survey spectra. Furthermore, signals coming from oxygen atoms both in O1s (Fig. 9b) and C1s (Fig. 9c) locations of relatively high intensity could be justified by the way how the excess reactant was removed. 
(a)

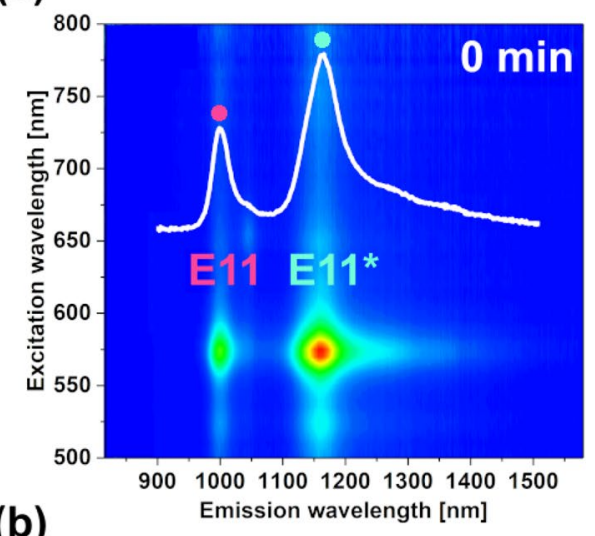

(b)
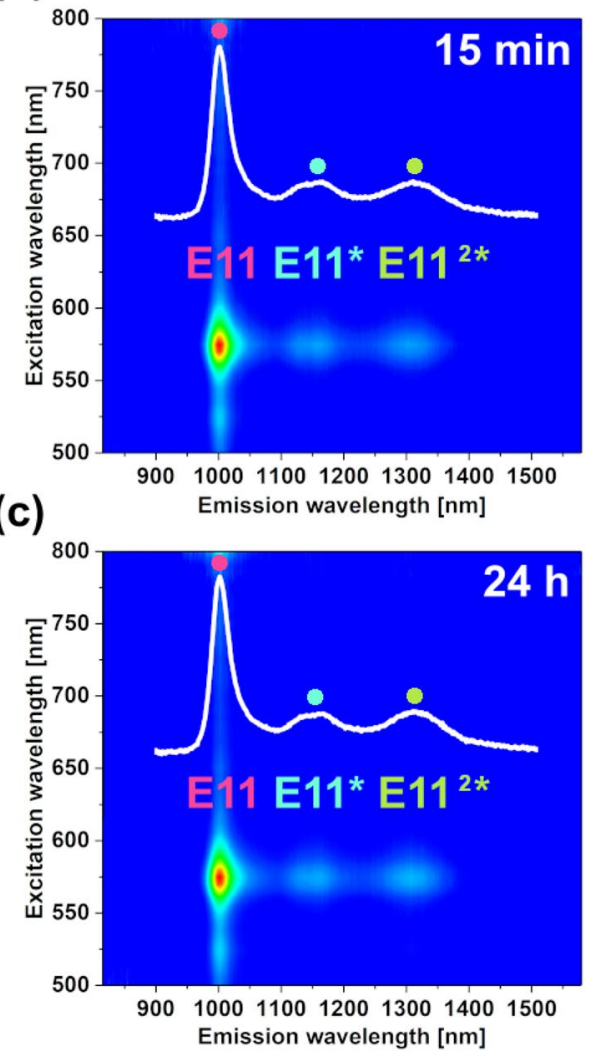

Figure 8. 2D PLE maps of (a) SWCNT dispersion after functionalization with BPO in toluene at $500 \mu \mathrm{g} / \mathrm{mL}$ concentration for $1 \mathrm{~h}$. Treatment of this sample with $\left[\mathrm{Et}_{4} \mathrm{~N}\right] \mathrm{OH}$ in methanol for (b) $15 \mathrm{~min}$ and (c) $24 \mathrm{~h}$. The insets show corresponding single PL spectra of SWCNTs collected at $575 \mathrm{~nm}$ excitation.

A septum was removed after $1 \mathrm{~h}$ of reaction time to evaporate this compound. The reaction mixture was then heated for $3 \mathrm{~h}$ in an open-system configuration under the influence of atmospheric oxygen. Because the solubility of oxygen in toluene increased with the rise in temperature ${ }^{40}$, we expected that in such configuration, the sample would become over-functionalized with oxygen groups. Nevertheless, the point of this experiment was to prove the presence of fluorine in the final product, which would indicate successful grafting of the surface, so this effect was disregarded. Based on the results presented above, we concluded that unambiguously the SWCNTs were modified by ester groups, which enhanced their light emission capabilities.

\section{Conclusions}

In summary, this contribution gave definite proof that the chemistry of organic radicals could be adapted to generate a new type of traps for mobile excitons in SWCNTs. The thermal dissociation of BPO gave benzoyloxy radicals, which then reacted with SWCNT to produce ester-modified SWCNTs. A range of methods validated the successful modification of the material this way. Firstly, it was found that the position of PL peaks from the functionalized material did not correspond with any of the results in the literature (oxidized, arylated or alkylated SWCNTs). Secondly, the grafted functional group was susceptible to hydrolysis upon exposure to the 
(a)

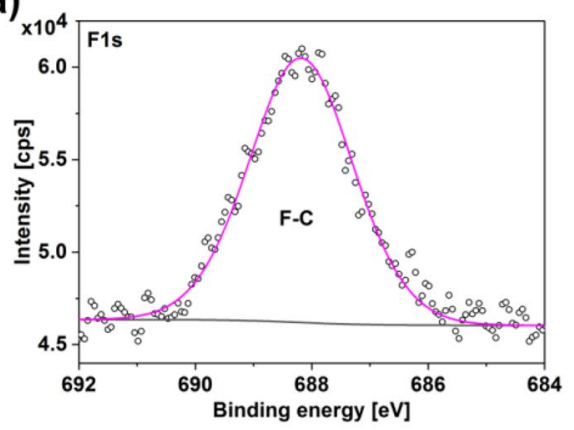

(b)

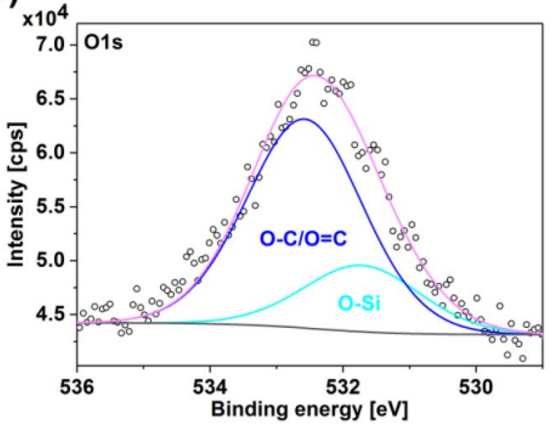

(c)

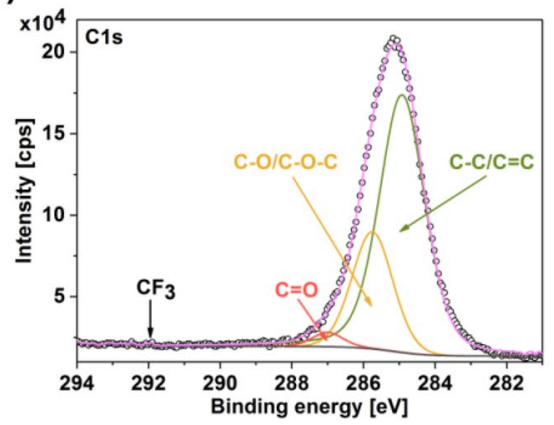

Figure 9. XPS spectrum of the SWCNT dispersion reacted with bis[4-(trifluoromethyl)benzoyl] peroxide recorded in the (a) F1s, (b) O1s, and (c) C1s areas. The O-Si peak comes from the substrate used for the deposition of the modified SWCNT material for analysis.

base as expected for ester functionality. Thirdly, fluorine-labeled BPO analog enabled us to detect fluorine on the SWCNT surface, a direct proof of successful modification by an organic group. In light of these results, we concluded that a benzoate derivative of SWCNTs was created.

The presented methodology combines the benefits of the established techniques of defect implantation in SWCNTs, while simultaneously circumvents their weak points. The transformation is simple to conduct and does not require dangerous chemicals as compared with typical reductive alkylations. Furthermore, in contrast to diazonium chemistry commonly employed for this purpose, it does not take days and does not involve lightsensitive chemicals. Instead, the process engages an oxidizing agent routinely used in organic chemistry, which successfully modifies the optical properties of SWCNTs in just an hour. Lastly, although it similarly quick as recently reported alternative techniques of oxidation, it also offers versatility because a spectrum of organic chemical groups can be attached to the surface via $\mathrm{C}-\mathrm{O}$ interconnect.

The key benefit of the proposed concept is that various polymers have been shown to have selectivity for the isolation of near-monochiral fractions of SWCNTs in organic solvents. By engaging the routine described herein, one can directly enhance the light emission capabilities of sorted SWCNTs without the need to carry out a surfactant exchange process to make the material water compatible. This approach, therefore, should pave a way towards the creation and application of a multitude of differently modified monochiral SWCNTs in photonics.

\section{Experimental}

Materials. Single-walled carbon nanotubes (SWCNTs) (CoMoCAT (6,5)-enriched), 2,2'-Azobis(2-methylpropionitrile) (AIBN), benzoyl peroxide (BPO), meta-chloroperoxybenzoic acid (mCPBA) $(\leq 77 \%)$, and $N$-iodosuccinimide (NIS) tetraethylammonium hydroxide solution $\sim 25 \%$ in methanol $(\sim 1.5 \mathrm{M})$ were purchased from 
Sigma-Aldrich. Bis[4-(trifluoromethyl)benzoyl] peroxide $\left(\mathrm{CF}_{3}-\mathrm{BPO}\right)$ was purchased from American Custom Chemicals Corporation. Toluene was purchased from Wako Pure Chemical Industries, Ltd. Poly(9,9-dioctylfluorenyl-2,7-diyl and bipyridine copolymer (PFO-Bpy) was obtained from American Dye Source, Inc. All chemicals were of analytical purity and used without further purification.

Preparation of SWCNT dispersion in toluene. A bath-type sonicator (BRANSON, CPX5800H-J), a tip-type sonicator (Tomy Seiko, UD-200), and an ultracentrifuge (Hitachi, Himac CS 100 GXL) were used for the preparation of the SWNT dispersion. In a $50 \mathrm{~mL}$ glass bottle, $3 \mathrm{mg}$ of the SWCNT material was dispersed in a toluene solution of PFO-Bpy $(0.4 \mathrm{mg} / \mathrm{mL}, 15 \mathrm{~mL})$ and sonicated using a bath-type sonicator for $3 \mathrm{~h}$ and then using a tip-type sonicator for $2 \mathrm{~h}$. The resulting dispersion was ultracentrifuged at $10,000 \mathrm{rpm}$ for $1 \mathrm{~h}$. The top $90 \%$ of the supernatant was collected for experiments.

Preparation of modified SWCNTs using radical organic reagents. Preliminary studies to select the reactant for the study. The reactions were carried out by combining the SWCNT dispersion obtained above and organic radical reagent solution (AIBN, BPO, $m \mathrm{CPBA}$, or NIS) to establish $80 \mu \mathrm{g} / \mathrm{mL}$ concentration of these radical-reservoirs in SWCNT toluene dispersion. Then, the reaction was allowed to proceed for $60 \mathrm{~min}$ at $100{ }^{\circ} \mathrm{C}$ under septum-sealed conditions. The products of the transformation were characterized by PL spectroscopy immediately after the reaction completion.

Kinetic studies using BPO. Time-dependence experiments were performed for $100 \mu \mathrm{g} / \mathrm{mL}$ concentration of BPO. The reactions were carried out by mixing the SWCNTs dispersion and an appropriate amount of BPO stock solution to reach $80 \mu \mathrm{g} / \mathrm{mL}$ in SWCNT toluene dispersion. The reaction was conducted for the specified time at $100{ }^{\circ} \mathrm{C}$ under septum-sealed conditions. The reaction course was monitored via PL spectroscopy at different time intervals $(15,30,45$, and $60 \mathrm{~min})$.

Concentration screening using BPO. The adjustment of the amount of BPO was necessary to obtain the optimum concentration of defects. We tested BPO concentrations from $1 \mu \mathrm{g} / \mathrm{mL}$ to $1200 \mu \mathrm{g} / \mathrm{mL}$ in SWCNT toluene dispersion at $100^{\circ} \mathrm{C}$. The reactions were carried out in the same way as described above $\left(100{ }^{\circ} \mathrm{C}, 60 \mathrm{~min}\right.$ process time). The reactions were monitored via PL spectroscopy after $60 \mathrm{~min}$.

Basic hydrolysis of modified SWCNTs. The obtained dispersions of modified-SWCNTs after exposure to $500 \mu \mathrm{g} /$ $\mathrm{mL}$ of BPO $(1 \mathrm{~mL})$ were treated with $2 \mu \mathrm{L}$ of a methanol solution of tetraethylammonium hydroxide $(1.5 \mathrm{M})$. The reactions were carried out by mixing the components at room temperature for the specified time. 2D PLE monitored the experiments in selected time intervals (15 min, and $24 \mathrm{~h}$ ).

Control reaction with $\mathrm{CF}_{3}$-BPO. SWCNT dispersion was exposed to $\mathrm{CF}_{3}-\mathrm{BPO}$ adjusted to reach the concentration of $100 \mu \mathrm{g} / \mathrm{mL}$ at $100{ }^{\circ} \mathrm{C}$ for $1 \mathrm{~h}$ under septum-sealed conditions, as previously described. After this time, the septum was removed, and $2 / 3$ of the reaction volume was evaporated by heating the mixture for another $3 \mathrm{~h}$. Trifluoromethylbenzene has a boiling point of $101-103{ }^{\circ} \mathrm{C}^{41}$, which is lower than that of toluene $111^{\circ} \mathrm{C}^{41}$, so it can be preferentially removed by evaporation. After the concentration step, the dispersion was deposited onto a p-type doped silicon substrate for XPS analysis.

Characterization. Optical properties of modified SWCNTs. Visible-near-infrared absorption (Vis/NIR, 400-1600 nm) and photoluminescence (PLE, excitation 500-800 nm, emission 815-1600 nm) spectra were measured using a V-670 (JASCO) and a HORIBA JOBIN YVON spectrofluorometer (FluorologR-3 with FluorEssence), respectively. Quartz cells with a 1-cm-path length were used for the optical measurements. The analysis was performed at room temperature. 2D PLE maps were normalized to the maxima of the peaks with the highest intensity. Raman spectra were measured using a Raman spectrometer (RAMANtouch, Nanophoton Corporation) at an excitation wavelength of $633 \mathrm{~nm}$ at room temperature.

Chemical composition of modified SWCNTs. X-ray photoemission spectra (XPS) were acquired using PREVAC EA15 hemispherical electron energy analyzer coupled with an X-ray source (PREVAC dual-anode XR40B, Al-Ka line, energy $1486.60 \mathrm{eV}$ ) as well as a $2 \mathrm{D}-\mathrm{MCP}$ detector. The measurements were conducted at the following conditions: $0.9 \mathrm{eV}$ scanning step, $200 \mathrm{eV}$ pass energy, and under $2 \times 10^{-8} \mathrm{~Pa}$ system base pressure. The binding energy (BE) scale was calibrated by positioning the reference peaks of $A u 4 f_{7 / 2}(84.0 \mathrm{eV})$. The obtained data were fitted using CASA XPS embedded algorithms as well as relative sensitivity factors. The background was modeled by the Shirley function and subtracted. The estimated uncertainty for the energy position of components is on the level of $0.1 \mathrm{eV}$.

Received: 17 August 2020; Accepted: 2 November 2020

Published online: 16 November 2020

\section{References}

1. Tans, S. J., Verschueren, A. R. M. \& Dekker, C. Room-temperature transistor based on a single carbon nanotube. Nature 393, 49-52 (1998). 
2. Kumanek, B. \& Janas, D. Thermal conductivity of carbon nanotube networks: A review. J. Mater. Sci. 54, 7397-7427 (2019).

3. Nanot, S., Hároz, E. H., Kim, J. H., Hauge, R. H. \& Kono, J. Optoelectronic properties of single-wall carbon nanotubes. Adv. Mater. 24, 4977-4994 (2012).

4. O'Connell, M. J. et al. Band gap fluorescence from individual single-walled carbon nanotubes. Science 297, 593-596 (2002).

5. Saha, A. et al. Narrow-band single-photon emission through selective aryl functionalization of zigzag carbon nanotubes. Nat. Chem. 10, 1089-1095 (2018).

6. Hertel, T., Himmelein, S., Ackermann, T., Stich, D. \& Crochet, J. Diffusion limited photoluminescence quantum yields in 1-D semiconductors: Single-wall carbon nanotubes. ACS Nano 4, 7161-7168 (2010).

7. Wei, X. et al. Photoluminescence quantum yield of single-wall carbon nanotubes corrected for the photon reabsorption effect. Nano Lett. https://doi.org/10.1021/acs.nanolett.9b04095 (2020).

8. Janas, D. Perfectly imperfect: A review of chemical tools for exciton engineering in single-walled carbon nanotubes. Mater. Horiz. 7, 2860-2881 (2020).

9. Wang, F., Dukovic, G., Brus, L. E. \& Heinz, T. F. The optical resonances in carbon nanotubes arise from excitons. Science 308, 838-841 (2005).

10. Piao, Y. et al. Brightening of carbon nanotube photoluminescence through the incorporation of sp 3 defects. Nat. Chem. 5, 840-845 (2013).

11. Setaro, A. et al. Preserving $\pi$-conjugation in covalently functionalized carbon nanotubes for optoelectronic applications. Nat. Commun. 8, 1-7 (2017).

12. Ghosh, S., Bachilo, S. M., Simonette, R. A., Beckingham, K. M. \& Weisman, R. B. Oxygen doping modifies near-infrared band gaps in fluorescent single-walled carbon nanotubes. Science 330, 1656-1659 (2010).

13. Lin, C. W. et al. Creating fluorescent quantum defects in carbon nanotubes using hypochlorite and light. Nat. Commun. 10, 666 (2019).

14. Powell, L. R., Piao, Y. \& Wang, Y. Optical excitation of carbon nanotubes drives localized diazonium reactions. J. Phys. Chem. Lett. 7, 3690-3694 (2016).

15. Shiraki, T., Shiraishi, T., Juhász, G. \& Nakashima, N. Emergence of new red-shifted carbon nanotube photoluminescence based on proximal doped-site design. Sci. Rep. 6, 1-9 (2016).

16. Powell, L. R., Kim, M. \& Wang, Y. Chirality-selective functionalization of semiconducting carbon nanotubes with a reactivityswitchable molecule. J. Am. Chem. Soc. 139, 12533-12540 (2017).

17. Coleman, K. S., Bailey, S. R., Fogden, S. \& Green, M. L. H. Functionalization of single-walled carbon nanotubes via the Bingel reaction. J. Am. Chem. Soc. 125, 8722-8723 (2003).

18. Maeda, Y., Takehana, Y., Yamada, M., Suzuki, M. \& Murakami, T. Control of the photoluminescence properties of single-walled carbon nanotubes by alkylation and subsequent thermal treatment. Chem Commun 51, 13462-13465 (2015).

19. Janas, D. Towards monochiral carbon nanotubes: A review of progress in the sorting of single-walled carbon nanotubes. Mater. Chem. Front. 2, 36-63 (2018).

20. Berger, F. J. et al. Brightening of long, polymer-wrapped carbon nanotubes by $\mathrm{sp}^{3}$ functionalization in organic solvents. ACS Nano 13, 9259-9269 (2019).

21. Maeda, Y. et al. Sonochemical reaction to control the near-infrared photoluminescence properties of single-walled carbon nanotubes. Nanoscale https://doi.org/10.1039/d0nr00271b (2020).

22. Zheng, Y., Bachilo, S. M. \& Weisman, R. B. Photoexcited aromatic reactants give multicolor carbon nanotube fluorescence from quantum defects. ACS Nano 14, 715-723 (2020).

23. Garrett, B. C. et al. Role of water in electron-initiated processes and radical chemistry: Issues and scientific advances. Chem. Rev. 105, 355-389 (2005)

24. Samanta, S. K. et al. Conjugated polymer-assisted dispersion of single-wall carbon nanotubes: The power of polymer wrapping. Acc. Chem. Res. 47, 2446-2456 (2014).

25. Graf, A. et al. Large scale, selective dispersion of long single-walled carbon nanotubes with high photoluminescence quantum yield by shear force mixing. Carbon 105, 593-599 (2016).

26. Ozawa, H., Ide, N., Fujigaya, T., Niidome, Y. \& Nakashima, N. One-pot separation of highly enriched $(6,5)$-single-walled carbon nanotubes using a fluorene-based copolymer. Chem. Lett. 40, 239-241 (2011).

27. Shiraishi, T., Shiraki, T. \& Nakashima, N. Substituent effects on the redox states of locally functionalized single-walled carbon nanotubes revealed by in situ photoluminescence spectroelectrochemistry. Nanoscale 9, 16900-16907 (2017).

28. Ju, S. Y., Doll, J., Sharma, I. \& Papadimitrakopoulos, F. Selection of carbon nanotubes with specific chiralities using helical assemblies of flavin mononucleotide. Nat. Nanotechnol. 3, 356-362 (2008).

29. Ju, S. Y., Kopcha, W. P. \& Papadimitrakopoulos, F. Brightly fluorescent single-walled carbon nanotubes via an oxygen-excluding surfactant organization. Science 323, 1319-1323 (2009).

30. Charles, E. \& Carraher, J. Polymer chemistry. Sci. Twentieth Cent. https://doi.org/10.4324/9781315079097-35 (2006).

31. Ni, S. et al. Sunlight-promoted cyclization versus decarboxylation in the reaction of alkynoates with N-iodosuccinimide: Easy access to 3-iodocoumarins. Green Chem. 18, 3935-3939 (2016).

32. $\mathrm{N}$-iodosuccinimide-promoted cascade trifunctionalization of alkynoates: Access to 1,1-diiodoalkenes. Organ. Lett.. https://doi. org/10.1021/acs.orglett.5b03685.

33. Ma, X. et al. Electronic structure and chemical nature of oxygen dopant states in carbon nanotubes. ACS Nano 8, 10782-10789 (2014).

34. He, X. et al. Tunable room-temperature single-photon emission at telecom wavelengths from $\mathrm{sp}^{3}$ defects in carbon nanotubes. Nat. Photonics 11, 577-582 (2017).

35. Shiraki, T., Uchimura, S., Shiraishi, T., Onitsuka, H. \& Nakashima, N. Near infrared photoluminescence modulation by defect site design using aryl isomers in locally functionalized single-walled carbon nanotubes. Chem. Commun. 53, 12544-12547 (2017).

36. Maeda, Y. et al. Frontispiece: Effect of substituents and initial degree of functionalization of alkylated single-walled carbon nanotubes on their thermal stability and photoluminescence properties. Chem. Eur. J. 23, 1789 (2017).

37. Ghosh, S., Bachilo, S. M., Simonette, R. A., Beckingham, K. M. \& Weisman, R. B. Oxygen doping modifies near-infrared band gaps in fluorescent single-walled carbon nanotubes. Science 330, 1656-1659 (2010).

38. Maeda, Y. et al. Effect of substituents and initial degree of functionalization of alkylated single-walled carbon nanotubes on their thermal stability and photoluminescence properties. Chem. Eur. J. 23, 1789-1794 (2017).

39. Shulga, Y. M. et al. XPS study of fluorinated carbon multi-walled nanotubes. J. Electron. Spectrosc. Relat. Phenom. 160, 22-28 (2007).

40. Wu, X. et al. Experimental investigation on the solubility of oxygen in toluene and acetic acid. Ind. Eng. Chem. Res. 53, 9932-9937 (2014).

41. Armarego, W. L. F. Purification of Laboratory Chemicals Eighth Edition. (2008).

\section{Acknowledgements}

D.J. and L.P. would like to thank the National Science Centre, Poland (under the OPUS program, Grant agreement 2019/33/B/ST5/00631), for financial support of the research. The authors also express gratitude to the 
National Agency for Academic Exchange of Poland (under the Academic International Partnerships program, grant agreement PPI/APM/2018/1/00004) for financial support of the internship at Kyushu University in Japan which enabled the execution of a part of the experimental work and publishing the results in the gold open access model. The authors acknowledge the ESpeFuM laboratory for access to the XPS experimental setup.

\section{Author contributions}

L.P. and D.J. conceived the research idea. L.P. conducted functionalization and characterization. M.K., Y.N., and H.A. participated in the characterization of the functionalized material. T.S. and D.J. supervised the work. L.P. and D.J. analyzed the data and prepared the manuscript. All authors reviewed the manuscript.

\section{Competing interests}

The authors declare no competing interests.

\section{Additional information}

Supplementary information is available for this paper at https://doi.org/10.1038/s41598-020-76716-9.

Correspondence and requests for materials should be addressed to D.J.

Reprints and permissions information is available at www.nature.com/reprints.

Publisher's note Springer Nature remains neutral with regard to jurisdictional claims in published maps and institutional affiliations.

(c) (i) Open Access This article is licensed under a Creative Commons Attribution 4.0 International License, which permits use, sharing, adaptation, distribution and reproduction in any medium or format, as long as you give appropriate credit to the original author(s) and the source, provide a link to the Creative Commons licence, and indicate if changes were made. The images or other third party material in this article are included in the article's Creative Commons licence, unless indicated otherwise in a credit line to the material. If material is not included in the article's Creative Commons licence and your intended use is not permitted by statutory regulation or exceeds the permitted use, you will need to obtain permission directly from the copyright holder. To view a copy of this licence, visit http://creativecommons.org/licenses/by/4.0/.

(c) The Author(s) 2020 